\title{
A PROOF OF AN INEQUALITY OF CARLEMAN
}

\section{LENNART CARLESON}

1. Let $m(x)$ be a convex function for $x \geqq 0$, satisfying $m(0)=0$. If $-1<p<\infty$, then the following inequality holds true:

$$
I_{1}=\int_{0}^{\infty} x^{p} e^{-m(x) / x} d x \leqq e^{p+1} \int_{0}^{\infty} x^{p} e^{-m^{\prime}(x)} d x=e^{p+1} I_{2} .
$$

Our argument yields that the sign of equality is excluded, but we shall not insist on this detail. The constant $e^{p+1}$ cannot be improved. To see this, we consider the function

$$
m(x)=\left\{\begin{array}{lr}
0, & 0 \leqq x \leqq 1, \\
(a+1) x \log x, & 1<x<\infty, a>p .
\end{array}\right.
$$

In this case, $I_{1}=(a-p)^{-1}+(p+1)^{-1}$ while $I_{2}=e^{-a-1}(a-p)^{-1}$ $+(p+1)^{-1}$. Since $a$ is any number greater than $p$, we see that $e^{p+1}$ in (1) cannot be replaced by any smaller number.

2. For the proof of (1), we choose a number $k>1$ and use the inequality

$$
m(k x) \geqq m(x)+(k-1) x m^{\prime}(x) .
$$

Hence

$$
k^{-p-1} \int_{0}^{A} x^{p} e^{-m(x) / x} d x
$$

$$
\begin{aligned}
& \leqq \int_{0}^{A} x^{p} e^{-m(k x) / k x} d x \leqq \int_{0}^{A} x^{p} e^{-m(x) / k x-((k-1) / k) m^{\prime}(x)} d x \\
& \leqq\left\{\int_{0}^{A} x^{p} e^{-m(x) / x} d x\right\}^{1 / k}\left\{\int_{0}^{A} x^{p} e^{-m^{\prime}(x)} d x\right\}^{(k-1) / k} .
\end{aligned}
$$

From this it follows when $A \rightarrow \infty$

$$
I_{1} \leqq k^{(p+1) k /(k-1)} I_{2} \text {. }
$$

As $k \rightarrow 1, k^{k /(k-!)} \rightarrow e$, and we obtain the desired inequality (1).

3. The case $p=0$ is known as Carleman's inequality: if $\sum_{1}^{\infty} a_{n}$ is a convergent series with positive terms, then

Received by the editors January 5, 1954. 


$$
\sum_{1}^{\infty}\left(a_{1} a_{2} \cdots a_{n}\right)^{1 / n}<e \sum_{1}^{\infty} a_{n}
$$

This follows immediately from (1). We observe that the series to the left attains its maximum if the terms of the given series are arranged in decreasing order. We then define $m(x)$ as the polygon passing through the points $\left(n, \sum_{1}^{n} \log \left(1 / a_{v}\right)\right), n=1,2, \cdots$. In the interval $(n-1, n), m^{\prime}(x)=\log \left(1 / a_{n}\right)$, while

$$
\frac{m(x)}{x} \leqq \frac{m(n)}{n}=\frac{1}{n} \sum_{1}^{n} \log \frac{1}{a_{\nu}} .
$$

As we cannot have equality in (4) for all $n$ and $x$, (3) follows.

UNIVERSITY OF UPPSALA 